\title{
Parâmetros acústicos de vozes de mulheres na pós-menopausa
}

\author{
Acoustic parameters of voices of women in post-menopause
}

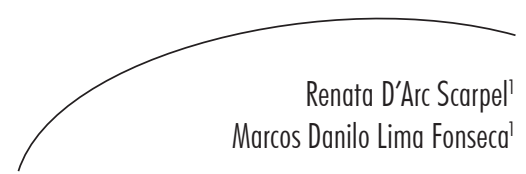

\section{Resumo}

Introdução: $\mathrm{O}$ envelhecimento da vOz caracteriza-se por alterações decorrentes do envelhecimento do corpo, interferindo na entonação vocal reveladora de estados da alma e do corpo. Investigar a qualidade vocal de mulheres na pós-menopausa por meio dos parâmetros objetivos torna-se importante para fomentar as pesquisas nessa área, bem como auxiliar na melhoria da qualidade de vida desse grupo. Objetivo: Verificar os valores dos parâmetros acústicos das vozes de mulheres no período de pós-menopausa. Método: Realizou-se estudo de caráter quantitativo e corte transversal com 23 mulheres em período de pós-menopausa, utilizando o software Praat 5.2.0. Os parâmetros considerados foram frequência fundamental, jitter, shimmer, proporção harmônico ruído e análise espectrográfica. A amostra foi composta por mulheres matriculadas numa Universidade Aberta da Terceira Idade em Salvador-BA. A análise dos dados foi descritiva, por porcentagem simples, e usou-se o teste $t$ para verificar a relação entre aumento de idade e piora vocal. Resultados: Apresentaram valores da frequência fundamental abaixo de $150 \mathrm{~Hz} 13 \%$ da amostra, e $34 \%$ entre 150 e $217 \mathrm{~Hz}$, demonstrando agravamento da voz. Observou-se significância estatística para piora desse parâmetro relacionado ao aumento da idade $(\mathrm{p}=0,02471)$. Houve diferença quanto aos parâmetros jitter e shimmer de acordo com o aumento da idade, porém sem significância estatística. Esses parâmetros acústicos estão relacionados a alterações no padrão vibratório das pregas vocais (ciclo vibratório). Os valores da proporção harmônico-ruído foram acima de 15.000 para todas as mulheres, mas também foram observados valores diminuídos com o aumento da idade, sem significância estatística. Conclusão: As alterações nos parâmetros acústicos observadas demonstram que as mulheres na pós-menopausa apresentaram mudanças no padrão vocal, o que pode levar à diminuição da qualidade de vida. Diante disso, necessita-se de um olhar mais atento quanto à necessidade da atuação fonoaudiológica nesse grupo.
Palavras-chave: Voz. Análise Acústica. Qualidade Vocal. Pós-menopausa. 


\section{Abstract}

Introduction: Voice aging is characterized by changes caused by body aging, interfering with the vocal intonations, revealing states of soul and body. To investigate the vocal quality of postmenopausal women using objective parameters is important to foster research in this area, as well as assisting in improving the quality of life of this group. Objective: To determine the acoustic parameters of the voice of women in postmenopause. Method: Quantitative cross-sectional study was conducted with 23 women in the postmenopausal period, using the Praat software 5.2.0. The parameters considered were fundamental frequency, jitter, shimmer, harmonic noise and spectrographic analysis. The sample consisted of women enrolled in the Open University of Third Age in Salvador-BA. Data analysis was descriptive, using percentages, and $t$ test to check the relationship between increasing age and vocal worsening. Results: In this sample, $13 \%$ had values below the fundamental frequency of $150 \mathrm{~Hz}$ and $34 \%$ between 150 and $217 \mathrm{~Hz}$, demonstrating voice deepening. There was a statistically significant worsening of this related to increasing age parameter $(\mathrm{p}=0.02471)$. There was difference between the jitter and shimmer parameters according to increasing age, but without statistical significance. These acoustic parameters are related to changes in the vibratory pattern of the vocal folds (vibration cycle). A harmonic noise ratio was above 15.000 in all women, but also had lower values with increasing age, with no statistical significance. Conclusion: The observed changes in acoustic parameters show that postmenopausal women had changes in vocal patterns, which can lead to decreased quality of life. Therefore, it requires a closer look at the need of speech therapy in this group.
Key words: Voice. Acoustic Analysis. Vocal Quality. Postmenopause.

\section{INTRODUÇÃO}

Cada vez mais, os idosos estão inseridos no mundo moderno. As mudanças sociais e econômicas influenciam diretamente a vida dessa população, e a expressão comunicativa de cada indivíduo é fator importante para adequação à nova realidade. Acompanhar esse desenvolvimento e tentar se inserir no contexto sócio-relacional, tendo voz ativa e coragem para isso, é um dos grandes desafios, o que faz com que esse novo modelo de vida sirva de estímulo e desafio para os idosos melhorarem sua qualidade de vida. Nesse contexto, a voz tem destaque fundamental como instrumento de comunicação, integração e indicador da qualidade de vida, o que propicia caminhos de equilíbrio psicossocial fundamentais para o idoso, permitindo que exerça sua individualidade, autonomia e cidadania. ${ }^{1}$

O desenvolvimento da voz "acompanha e representa o desenvolvimento do indivíduo, tanto do ponto de vista físico, como psicológico e social". ${ }^{2}$ O envelhecimento da voz, chamado de presbifonia, caracteriza-se por alterações no processo vocal decorrentes do envelhecimento do corpo, influenciando nas entonações vocais, reveladoras de estados da alma e do corpo. ${ }^{3,4}$

Boulet \& Oddens ${ }^{5}$ relatam que na pósmenopausa ocorrem mudanças na voz, que podem ser decorrentes do impacto do hipoestrogenismo sobre a laringe. Os autores constataram, em esfregaços das pregas vocais, que os tecidos se mostraram atróficos e semelhantes àqueles do colo uterino. Este estudo, realizado em 100 mulheres após a menopausa, constatou expressivas alterações laríngeas e vocais. Os achados de Brunings et al. ${ }^{6}$ também indicaram que a quantidade de estrógeno e progesterona na laringe de mulheres idosas pode ter relação com edema nas pregas vocais.

Os sintomas de agravamento da voz, a redução de microestabilidade vocal e de variações de tons podem ocorrer na pós-menopausa 
em concomitância com calorões, problemas cardiovasculares, incontinência urinária, osteoporose, depressão e demência. ${ }^{7.8}$ A laringe, com o avançar da idade, também sofre alterações nos aspectos morfológicos, podendo aumentar e exibir fibrose da musculatura envolvida, resultando em redução da mobilidade da estrutura da prega vocal e prejuízo na qualidade vocal. ${ }^{9-12}$

D'haeseleer et al. ${ }^{13}$ encontraram, na avaliação perceptivo-auditiva da qualidade vocal de mulheres na pós-menopausa, diferenças quanto ao grau de disfonia, principalmente quanto a soprosidade e tensão vocal, comparadas às mulheres na pré-menopausa. $\mathrm{Na}$ análise acústica vocal computadorizada, também foram encontradas diferenças estatisticamente significantes nesses dois grupos quanto a faixa de frequência e parâmetros acústicos (frequência fundamental, variação da frequência fundamental, índices de alteração de tremor vocal).

Dessa forma, por oferecer uma compreensão diagnóstica mais objetiva, a análise acústica vocal computadorizada é um grande avanço na área da voz. Tal procedimento, associado aos avanços na compreensão da fisiologia vocal e ao desenvolvimento científico, ${ }^{14}$ possibilita ao clínico uma riqueza de dados objetivos e quantificados, os quais auxiliam na compreensão do mecanismo da fonação e, consequentemente, permitem descrever quase por completo a voz humana. ${ }^{15,16}$

A análise acústica da voz fornece dados normativos ou de base para realidades vocais distintas. Apesar de uma grande quantidade de informações referentes aos parâmetros vocais serem fornecidas pela análise acústica, esse método de análise ainda é pouco conhecido e explorado. Entre as medidas acústicas oferecidas pelos laboratórios de vOz, as principais que apresentam aplicação clínica são: frequência fundamental, medidas de intensidade, medidas de ruído e medidas de perturbação da frequência e da intensidade. $^{17}$

A frequência fundamental (f0) é a velocidade na qual uma forma de onda se repete por unidade de tempo, o que é indicado por $\mathrm{Hz}$ (Hertz).
A f0 da voz de um indivíduo é o reflexo das características biodinâmicas das pregas vocais e de sua integração com a pressão subglótica, sendo afetada pelo sexo e pela idade. ${ }^{2}$ É determinada fisiologicamente pelo número de ciclos que as pregas vocais fazem em um segundo, ou seja, pelo número de ciclos glóticos que se repetem. Portanto, qualquer ajuste que reduza os ciclos glóticos vai reduzir também a frequência fundamental. $^{18}$

Quanto à f0 no gênero feminino, constata-se diminuição nesse valor a partir dos 50 anos de idade, quando se inicia o processo do climatério, em que, além da voz, ocorrem mudanças como o aumento do peso corporal. Após os 60 anos, ocorre o início da senescência da voz. ${ }^{19}$

D'haeseleer et al., ${ }^{20}$ no intuito de investigar a relação entre índice de massa corporal e alteração na frequência fundamental de mulheres na pósmenopausa, avaliaram 105 mulheres, sendo que 41 estavam na pré-menopausa, 26 na pósmenopausa sem reposição hormonal e $26 \mathrm{com}$ reposição hormonal. Seus resultados mostraram correlação positiva $(\mathrm{p}=0,021)$ entre aumento da massa corporal e diminuição da frequência fundamental em mulheres pós-menopausa sem reposição hormonal.

O jitter consiste em uma medida da irregularidade percentual na altura da nota vocal (perturbação da f0), enquanto o shimmer é uma medida da irregularidade percentual na amplitude da nota vocal, sendo muitas vezes considerada como a perturbação da amplitude. O shimmer, portanto, mede a variação na intensidade dos ciclos adjacentes de vibração das cordas vocais e modifica-se com a redução da resistência glótica e lesões de massa nas cordas vocais, estando correlacionado com a presença de soprosidade ou ruído à emissão.?

A proporção harmônico-ruído, conhecida em inglês como barmonic-to-noise-ratio (HNR), contrasta com o sinal regular das pregas vocais e do trato vocal, oferecendo um índice que relaciona o componente harmônico versus o componente ruído da onda acústica. ${ }^{18}$ Desde 
que foi criada, a HNR foi considerada um dos melhores parâmetros de aplicação clínica, tanto na quantificação dos desvios vocais como na avaliação dos procedimentos dos tratamentos das disfonias, apresentando relação direta com a qualidade vocal (rouquidão). Atualmente, a HNR é facilmente obtida nos programas acústicos computadorizados de voz. ${ }^{18}$

Frente à nova realidade de vida para o idoso, é fundamental investigar os parâmetros de medidas acústicas que caracterizam o período de pós-menopausa, uma vez que influenciam diretamente na elaboração dos tratamentos fonoaudiológicos na área de voz. A depender das alterações vocais encontradas, diferentes são os enfoques da reabilitação fonoaudiológica. Assim, com esse conhecimento mais específico, é possível promover mudanças na elaboração de programas de reabilitação vocal que sejam mais eficientes (como por exemplo, diminuição da rouquidão ou soprosidade), ou no aprimoramento da voz (como por exemplo, ajuste da frequência fundamental ou da intensidade vocal), contribuindo para o aumento da qualidade de vida dessa população.

Dessa forma, o objetivo deste trabalho foi avaliar as medidas acústicas das vozes de mulheres na pós-menopausa, verificando aspectos da qualidade vocal, por meio da medição da frequência fundamental e seus índices de perturbação de frequência e intensidade (jitter e shimmer), assim como análise da proporção harmônico-ruído e análise espectrográfica.

\section{METODOLOGIA}

Foi realizado estudo de caráter quantitativo e corte transversal. A coleta dos dados foi realizada entre os meses de junho e julho de 2013. Alunas da Universidade da Terceira Idade (UATI), em Salvador-BA, foram convidadas a participar do estudo e orientadas sobre os procedimentos aos quais seriam submetidas. Receberam informações orais e escritas sobre o objetivo do trabalho, bem como de todos os aspectos éticos que regem as pesquisas com seres humanos.

A amostra foi aleatória e participaram do estudo 23 das 30 mulheres que se encontravam matriculadas na universidade. Os critérios de inclusão foram: mulheres em período de pósmenopausa (acima dos 55 anos de idade), sem a utilização de reposição hormonal, não fumantes, que não apresentavam queixa de rouquidão prévia à menopausa e que aceitaram participar da pesquisa. As participantes foram divididas em três grupos para análise: 60 a 70 anos, 71 a 80 anos e com mais de 80 anos.

Os registros acústicos foram coletados com microfone profissional unidirecional modelo Headset multimídia da marca Philips, para tornar mais eficaz a coleta das vozes, evitando diminuição do parâmetro de intensidade vocal por afastamento da boca em relação ao microfone. As vozes foram gravadas, individualmente, em um ambiente silencioso, com as participantes devidamente sentadas. Solicitou-se a emissão sustentada da vogal /e/ e a contagem de um até dez.

Para esta pesquisa, foi utilizado o programa Praat 5.2.0, com análise das seguintes medidas acústicas: frequência fundamental, medidas de perturbação de jitter e shimmer e proporção harmônico-ruído. Também houve análise das espectrografias relacionadas às vozes, realizadas por dois juízes fonoaudiólogos, que atuam na área da voz com análise acústica computadorizada.

Como o programa Praat 5.2.0 analisa no máximo dez segundos de emissão, optou-se por analisar os primeiros dez segundos de cada gravação. $\mathrm{Na}$ comparação dos resultados dos parâmetros acústicos para a emissão do /e/ longo e contagem de um a dez, foram encontrados valores similares, assim optou-se por descrever apenas os resultados da vogal prolongada. 
A análise dos dados foi de forma descritiva, por meio de porcentagem simples. Utilizou-se também o teste $t$ para verificar se havia diferença estatística entre a piora dos parâmetros acústicos e o aumento da idade.

Todas as etapas metodológicas do presente artigo foram realizadas conforme os princípios contidos na Declaração de Helsinki, assim como as orientações da Resolução $n^{\circ} 466 / 2012$, do Conselho Nacional de Saúde (CNS), que aprova as diretrizes e normas regulamentadoras de pesquisas envolvendo seres humanos. Todas as participantes, além da assinatura do Termo de Consentimento Livre e Esclarecido, concordaram com a divulgação dos resultados por meio de artigo científico. A pesquisa for aprovada pelo Comitê de Ética em Pesquisa da Universidade do Estado da Bahia, com parecer nº 276.861/2013.

\section{RESULTADOS}

Entre as 23 participantes, 52,17\% tinham entre 60 e 70 anos, 39,13\% entre 70 e 80 anos e $8,69 \%$ acima de 80 anos.

Dos valores encontrados quanto à frequência fundamental (f0), apenas três das 23 vozes, equivalentes a $13 \%$, apresentaram-se abaixo de $150 \mathrm{~Hz}$ (figura 1). Desse total, duas mulheres tinham entre 71 e 80 anos, e uma, acima de 80 anos. Não foram encontrados valores abaixo de $150 \mathrm{~Hz}$ no grupo de mulheres entre 60 e 70 anos.

Além dos resultados citados, verificou-se que oito vozes, $34,78 \%$, apresentaram $\mathrm{f} 0 \mathrm{com}$ valores entre 150 e $170 \mathrm{~Hz}$ (figura 2). Deste total, cinco mulheres tinham entre 60 e 70 anos, duas entre 70 e 80 e uma acima de 80 anos.

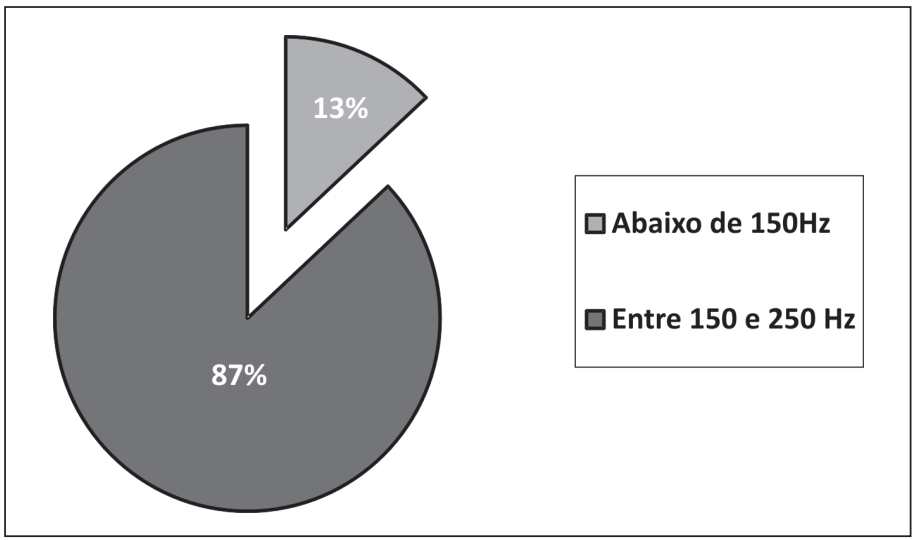

Figura 1. Distribuição quanto à frequência fundamental abaixo de $150 \mathrm{~Hz}$ de mulheres na pósmenopausa. Salvador-BA, 2013.

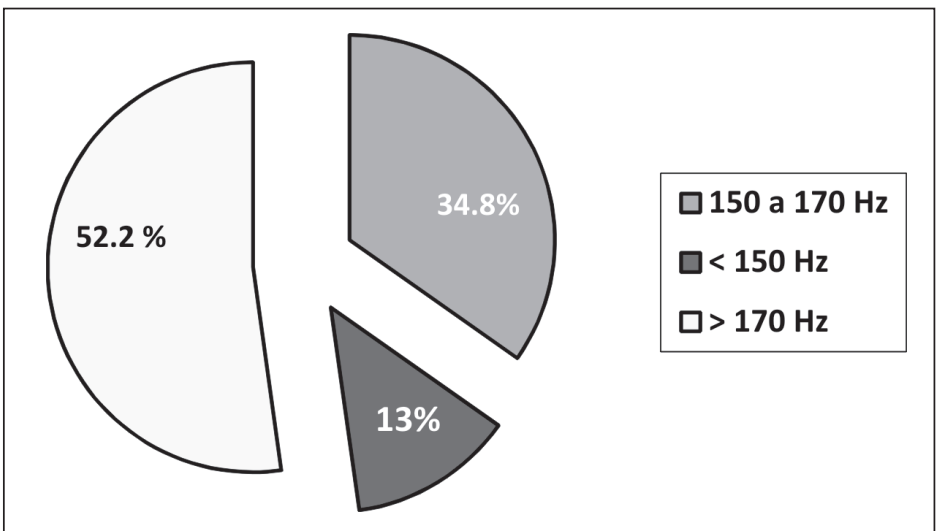

Figura 2. Distribuição quanto à frequência fundamental (f0) de mulheres na pós-menopausa. SalvadorBA, 2013. 
Cruzando os valores de f0 de acordo com as diferentes idades, observou-se significância estatística com $\mathrm{p}=0,02471$ (tabela 1), ou seja, com o aumento da idade existe um aumento do agravamento da voz, podendo chegar à faixa de normalidade masculina.

As médias dos valores de jitter local encontrados para emissão do /e/ longo foram: 0,422 no grupo de mulheres entre 60 e 70 anos; 0,783 no grupo entre 71 e 80 e 0,783 no grupo acima de 80 anos (tabela 1). Apesar de no teste estatístico não ter havido piora do parâmetro com o aumento da idade ( $\mathrm{p}=0,26763)$, observase que acima de 70 anos houve aumento dos valores das médias.
Para o parâmetro shimmer local, as médias dos resultados de acordo com a idade estão demonstradas na tabela 1. No grupo de mulheres entre 60 e 70 anos, a média foi 2,406; no grupo entre 71 e 80 anos, foi 3,350 e nas mulheres acima de 80 anos, 3,573. Novamente não houve significância para piora desse parâmetro de acordo com a idade $(\mathrm{p}=0,34245)$, apesar de verificar-se que no primeiro grupo os valores foram bem diferentes que nos grupos seguintes.

$\mathrm{Na}$ análise do parâmetro $\mathrm{HNR}$, as médias dos valores a partir da emissão do /e/ longo, em todos os grupos foi acima de 15,300 (tabela 1). Apesar disso, buscou-se verificar se haveria piora dessa relação com o aumento da idade. Os resultados não mostraram significância estatística para tal fato $(p=0,336972)$.

Tabela 1. Distribuição quanto às médias dos valores da frequência fundamental (f0), jitter, shimmer e proporção harmônico-ruído (HNR), de acordo com a idade de mulheres na pós-menopausa. SalvadorBA, 2013.

\begin{tabular}{ccccc}
\hline & 60 a 70 anos & 71 a 80 anos & Acima de 80 anos & P valor \\
\hline f0 & $182,16 \mathrm{~Hz}$ & $168,86 \mathrm{~Hz}$ & $148,69 \mathrm{~Hz}$ & $0,02471^{*}$ \\
Jitter & 0,422 & 0,782 & 0,783 & 0,26763 \\
Shimmer & 2,406 & 3,350 & 3,573 & 0,34245 \\
HNR & 18,917 & 15,955 & 15,300 & 0,336972 \\
\hline
\end{tabular}

$* \mathrm{p}<0,05=$ estatisticamente significante

\section{DISCUSSÃO}

Frente às poucas publicações quanto aos parâmetros acústicos e valores de normalidade em vozes de mulheres na pós-menopausa, sobretudo na literatura nacional com $\mathrm{O}$ software Praat 5.2.0, optou-se por discutir conceitualmente os resultados com base em pesquisas existentes sobre o assunto, mesmo que com softwares diferentes.
Behlau et al. ${ }^{21}$ afirmam que a frequência fundamental (fo) é um importante parâmetro na avaliação anatômica e funcional da laringe e é determinada pelo número de ciclos que as pregas vocais realizam por segundo. Essa medida é o resultado da interação entre o comprimento, a massa e a tensão das pregas vocais durante a fonação.

Entre os parâmetros acústicos, a f0 tem se mostrado o mais consistente entre diferentes 
sistemas de análise acústica. Os valores de normalidade para o sexo feminino se encontram entre 150 e $250 \mathrm{~Hz} ;{ }^{21}$ assim, os valores de anormalidade da f0, para emissão do /e/ longo encontrados nesta pesquisa, estão abaixo do limite de normalidade de $150 \mathrm{~Hz}$ indicados por esses autores. ${ }^{21}$

Embora os valores de f0 compreendidos entre 150 e $170 \mathrm{~Hz}$ estejam dentro do limite de normalidade, por ter proximidade com a faixa de frequência correspondente ao sexo masculino, vozes dentro dessa faixa indicam uma tendência ao agravamento vocal para as mulheres. Sendo assim, a soma dos resultados alterados (13\%) para f0 (figura 1) abaixo de $150 \mathrm{~Hz}$, ou seja, dentro do valor de normalidade para a voz masculina, ${ }^{19}$ com os resultados dos valores compreendidos entre 150 e $170 \mathrm{~Hz}(34,78 \%$ ) (figura 2), totaliza $47,78 \%$, e demonstra grande quantidade de vozes com características de agravamento vocal.

Os resultados encontrados para f0 estão de acordo com as postulações de alguns autores. Raj et al. ${ }^{22}$ encontraram agravamento nas vozes de mulheres pós-menopausa (valores médios de $204 \mathrm{~Hz}$ ), comparadas ao grupo controle (valores médios de $232,06 \mathrm{~Hz}$ ) com significância estatística $(p=0,00)$. D'haeseleer et al., ${ }^{13}$ comparando vozes de mulheres na pré-menopausa com mulheres na pós-menopausa, também encontraram valores médios na pós-menopausa de $116,35 \mathrm{~Hz}$, com diferença estatística significativamente $(p=0,007)$. Autores relatam que essas mudanças fazem com que as vozes femininas se pareçam com as masculinas e isso gere perda de parte da possibilidade de identificação referente ao sexo do falante. ${ }^{1,23}$

Outros autores referem que o agravamento vocal de mulheres idosas pode ser consequência das mudanças fisiológicas que ocorrem na laringe com o aumento da idade. ${ }^{3,24}$ A diminuição da f0 foi atribuída por alguns autores ao alto índice de edema da mucosa das pregas vocais, devido às alterações pós-menopausa. ${ }^{25,26}$ Nesta pesquisa, houve significância estatística para o aumento da idade relacionado à diminuição da f0.
As medidas de perturbação ciclo a ciclo avaliam as variações do sinal acústico, ou seja, estão relacionadas ao quanto determinado período de vibração glótica se diferencia do outro que o sucede, com relação à frequência (jitter) e à intensidade (shimmer). É importante destacar que os resultados das medidas de jitter e shimmer dependem dos métodos utilizados por cada programa e podem variar de acordo com idade, sexo e vogal utilizada, não havendo ainda padronização para essas medidas. ${ }^{17,27,28}$

O jitter, que se refere à perturbação ciclo a ciclo da frequência da voz, ${ }^{18,29}$ é uma medida objetiva, que avalia pequenas irregularidades dos pulsos glóticos, refletindo a rouquidão da $\mathrm{voz}^{30}$ ou o ruído da voz. As medidas de shimmer refletem a perturbação da amplitude ciclo a ciclo, e seu aumento está relacionado à diminuição ou inconsistência do coeficiente de contato das pregas vocais. Além disso, podem ser relacionadas à presença de soprosidade (escape de ar não sonorizado) na voz ou ao ruído como um todo. ${ }^{31}$

As médias dos resultados para os índices de perturbação, respectivamante, jitter e shimmer, demonstram que, apesar da não significância estatística (tabela 1), houve aumento dos valores de acordo com a idade. Acredita-se que isso possa ter relação com uma possível piora da qualidade vocal, com presença de rouquidão e soprosidade nas vozes dos grupos de mulheres de 71 a 80 anos e mais de 80 anos.

É aceitável que qualquer voz seja, de certa forma, instável em face de fatores de ordem neurológica, emocional e biomecânica. É, por isso, previsível a presença de um pequeno grau de perturbação e irregularidade no sinal vocal..$^{25}$ Para se refletir melhor sobre os dados, seria necessária a avaliação laringológica da amostra, fato que não foi previsto para esta pesquisa, mas que poderia complementar a discussão.

A proporção harmônico-ruído (HNR) contrasta com o sinal regular das pregas vocais e do trato vocal, oferecendo um índice que relaciona o componente harmônico versus o componente ruído da onda acústica. ${ }^{18}$ Assim, essa medica avalia 
a presença de ruído no sinal de voz analisado, apresentando relação direta com a qualidade vocal, determinando a percepção geral de ruído e de rouquidão no sinal vocal. Portanto, quanto maior for HNR, melhor será a qualidade vocal. ${ }^{32}$

Dentre as médias dos valores de HNR nas vozes coletadas nesta pesquisa, os valores foram cima de $15 \mathrm{~dB}$ (tabela 1). Raj et al., ${ }^{22}$ em seu estudo, encontraram valores do HNR similares para melhores na pós-menopausa $(19,28 \mathrm{~dB}) \mathrm{em}$ comparação ao grupo controle $(19,94 \mathrm{~dB})$. Podese supor, com isso, que as alterações laríngeas decorrentes da idade não tenham a capacidade de perturbar a produção dos harmônicos, como acontecem com as vozes com patologias laríngeas. Além disso, infere-se que as alterações vocais decorrentes da idade não levem a alterações desse parâmetro, mas seria necessário o aumento do número amostral, assim como outras pesquisas semelhantes para a comprovação desse achado.

Por fim, outra forma de avaliação acústica é a espectrografia, que pode ser definida como um gráfico que mostra a intensidade por meio do escurecimento ou coloração do traçado, as faixas de frequência no eixo vertical e o tempo no eixo horizontal. Sua representação mostra estrias horizontais denominadas harmônicos. O espectrograma demonstra visualmente as características acústicas. ${ }^{33}$

Nesta pesquisa foram observadas, a partir da espectrografia acústica, evidências de instabilidade vocal referente à intensidade, por meio do grau do escurecimento do traçado acústico que não se mantiveram constantes ao longo da emissão da vogal sustentada /e/, principalmente do meio para o final das emissões. Isso foi verificado em $56,52 \%$ das amostras analisadas.

Pontes $^{34}$ afirma que a coloração ou escurecimento dos harmônicos representa a intensidade do sinal acústico, a qual está relacionada à intensidade da voz, que por sua vez depende da resistência glótica e da tonicidade laríngea. ${ }^{22,35}$ Ocorreram variações em torno da regularidade geral do traçado e presença de interrupções em momentos isolados em torno da emissão. Também foram encontradas modulações leves em torno do traçado da frequência dos harmônicos, evidenciando presença de instabilidade durante a emissão vocal em 65,21\% das amostras.

Por fim, como descrito acima, verificouse que em mulheres de 60 a 70 anos, todos os parâmetros acústicos foram melhores que em mulheres mais velhas, acima de 71 anos, cujos valores foram similares. Ainda assim, não é possível evidenciar esses resultados, já que como limitação desta pesquisa, a amostra foi reduzida, não houve avaliação laringológica para comparar os resultados à imagem laríngea e são poucos os estudos na literatura com a utilização do software Praat, principalmente quanto aos valores de normalidade dos parâmetros acústicos analisados.

\section{CONCLUSÃO}

Os resultados encontrados para frequência fundamental indicaram valores abaixo ou próximo de $150 \mathrm{~Hz}$, indicando agravamento das vozes nessa amostra. Verificou-se também que as mulheres com idade entre 70 e 80 anos e acima de 80 anos apresentaram piora em relação aos parâmetros acústicos analisados, com significância estatística apenas para a relação entre aumento de idade e agravamento vocal. Quanto à análise espectrográfica, foram observadas instabilidade de frequência e intensidade na maior parte da amostra.

Por ter sido observada escassa literatura, principalmente com a utilização do software Praat, acredita-se ser necessária a produção de novos estudos voltados para a análise acústica da voz de mulheres na pós-menopausa associada à avaliação laringológica, e que enfatizem 
também o parâmetro "instabilidade" por meio da análise espectrográfica, já que as mudanças no padrão vocal podem levar à diminuição da qualidade de vida. Diante disso, sugere-se um olhar mais atento quanto à necessidade da atuação fonoaudiológica nesse grupo.

\section{REFERÊNCIAS}

1. Filho LFB. O processo de envelhecimento e o comportamento vocal. Monografia de conclusão curso de especialização em voz. Rio de Janeiro: Cefac, 1999.

2. Prado SD, Sayd JD. A pesquisa sobre envelhecimento humano no Brasil: grupos e linhas de pesquisa. Ciência \& Saúde Coletiva. 2004; 9(1):57-67.

3. Behlau MS. Voz: o livro do especialista. v. 2. Rio de Janeiro: Revinter; 2005.

4. Costa HO, Matias C. O impacto da voz na qualidade da vida da mulher idosa. Rev. Bras Otorrinolaringol. 2005; 71(2):172-8.

5. Boulet MJ, Oddens BJ. Female voice changes around and after the menopause - an initial investigation. Maturitas 1996:23:15-21.

6. Brunings JW, Schepens JJBFG, Peutz-Kootstra CJ, Kross KW. The expression os estrogen and progesterone receptors in the human larynx. J Voice 2013; 27(3): 376-80.

7. Abitbol J, Abitbol P, Abitbol B. Sex hormone and the female voice. J Voice 1999; 3: 424-46

8. Borker SA, Venugopalan PP, Bhat SN. Study os menopausal symptoms, and perceptions about menopause among women at a rural community in Kerala. J Midlife Health 2013; 4(3):182-7

9. Soyama CK, Espassatempo CL, Gregio FN, Camargo Z. Qualidade vocal na terceira idade: parâmetros acústicos de longo termo de vozes masculinas e femininas. Rev CEFAC 2005; 7(2):267-79.

10. Casiano RR, Ruiz PJ, Goldstein W. Histopathologic changes in the aging human cricoarytenoid joint. Laryngoscope. 1994; 104(5):533-8.

11. DeBiase NG, Cervantes O, Abrahão. A voz no idoso. Acta Awho 1998; 17(2):70-2.

12. Ferreira LM. Aprimoramento vocal na terceira idade. In: Pinho SMR. Fundamentos em fonoaudiologia: tratando dos distúrbios da voz. Rio de Janeiro: Guanabara Koogan; 1998. p. 115-8.

\section{AGRADECIMENTOS}

Agradecimento especial às alunas da Universidade da Terceira Idade, por terem se disponibilizado a gravar suas vozes para a pesquisa.

13. D'haeseleer E, Depypere H, Claeys S, Wuyts FL, De Ley S, Lierde KMV. The impact os menopause on vocal quality. Menopause 2011;18(3):267-72

14. Corazza VR, Silva VFC, Queija DS, Dedivits RA, Barros, APB. Correlação entre achados estroboscópicos, perceptivo-auditivos e acústicos em adultos sem queixa vocal. Rev. Bras. Otorrinolaringol. 2004;70(1):30-4.

15. Araújo SA, Grellet M, Pereira JC, Rosa MO. Normatização de medidas acústicas da voz normal. Rev. Bras. Otorrinolaringol; 2002;68(4):540-4.

16. Santos IR. Análise acústica da voz de indivíduos na terceira idade [dissertação]. São Carlos:Universidade de São Paulo (USP);2005.

17. Barros APB, Carrara-De Angelis E. Análise acústica da voz. Em:Dedivits RA, Barros APB. Métodos de avaliação e diagnóstico de laringe e voz. São Paulo: Louvise; 2002. p. 201-21.

18. Behlau M, Madazio G, Feijó D, Pontes P. Avaliação de Voz. Em: Behlau M. Voz: o livro do especialista I. Rio de Janeiro: Revinter; 2001.

19. Polido AM, Martins MASUR, Hanayama EM. Percepção do envelhecimento vocal na terceira idade. Rev. CEFAC; 2005; 7(2):241-51.

20. D'haeseleer E, Depypere H, Claeys S, Lierde KMV. The relation between body mass index and speaking fundamental frequency in premenopausal and postmenopausal women. Menopause 2011;18(7):754-8.

21. Behlau MS, Tosi O, Pontes P. Determinação da frequência fundamental e suas variações em altura (Jitter) e intensidade (Shimmer) para falantes do português brasileiro. Acta AWHO; 1985; 4:5-9.

22. Raj A, Gupta B, Chowdhury A, Chadha S. Study of voice changes in various phases menstrual cycle and in postmenopausal womem. J Voice 2010; 24(3):363-8.

23. Cassol M, Behlau M. Análise percetiva-auditiva e acústica da voz de indivíduos idosos pré e pós intervenção fonoaudiológica. Fonoaudiol Brasil. 2000; 3(4):32-44. 
24. Feijó A, Estrela F, Scalco M. Avaliação perceptiva e quantitativa da voz na terceira idade. Fonoaudiol. Brasil; 1998; 1(1):22-31.

25. Honjo I, Isshiki N. Laryngoscopic and voice characteristics of aged persons. Arch Otolaryngol; 1980; 106(3):149-50.

26. Morsomme D. Presbyphonia: voice differences between the sexes in the elderly: comparison by maximum phonation time, phonation quotient and spectral analysis. Logop Phoniatr Vocol; 1997; 22(1):9-14.

27. Santos I. Análise acústica da voz de indivíduos da terceira idade [dissertação]. Universidade de São Carlos; 2005

28. Andrade LMO. Determinação dos limiares de normalidade dos parâmetros acústicos da vOz [dissertação]. São Carlos: Universidade de São Paulo (USP); 2003.

29. Oguz H, Tarhan E, Korkmaz M, Yilmaz U, Safak MA, Demirci M, et al. Acoustic analysis findings in objective laryngopharyngeal reflux patients. J Voice; 2007; 21(2):203-10.
30. Kandagan T, Seifert E. Influence of aging and sex on voice parameters in patients with unilateral vocal cord paralysis. Laryngoscope; 2005;115:655-60.

31. Finger LM, Cielo CA, Schwarz K. Medidas vocais acústicas de mulheres sem queixas de voz e com laringe normal. Braz. j. otorhinolaryngol. (Impr.) vol.75 no.3 São Paulo May/June 2009

32. Meurer EM et al. Voz e fala no menacme e na pósmenopausa. Rev. Bras. Saúde Matern. Infant., Recife, 4 (3): 281-286, jul. / set., 2004.

33. Valentim AF, Côrtes MG, Gama ACC Analise espectrográfica da voz: efeito do treinamento visual na confiabilidade da avaliação. Rev Soc Bras Fonoaudiol. 2010;15(3):335-42

34. Pontes PAL, Vieira VP, Gonçalves MIR, Pontes AAL. Características das vozes roucas, ásperas e normais: análise acústica espectrográfica comparativa. Rev. Bras. Otorrinolaringol; 2002; 68(2):182-8.

35. Drumond LB, Gama ACC. Correlação entre dados espectrográficos e perceptivo-auditivos de vozes disfônicas. Fono atual; 2006;35:49-58. 